\title{
Influencia de la ocupación en actividades de la vida diaria y de ocio sobre la salud real de la persona mayor
}

\section{José M. AGUILAR-PARRA*¹, Remedios LÓPEZ-LIRIA*, José M. FERNÁNDEZ-

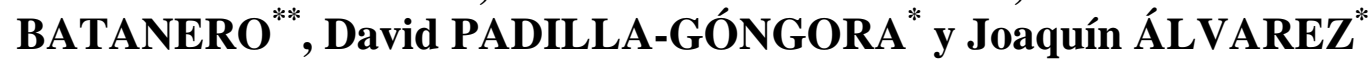

(*) Universidad de Almería, España (**) Universidad de Sevilla, España.

(Recibido 2 Marzo, 2016; Aceptado 10 Mayo, 2016)

RESUMEN: El siglo XXI está destinado a conformarse por una población con tendencia a envejecer. Por esto, se han de buscar estrategias para mejorar la calidad de vida de este sector de población. La variable que incide más determinantemente en la calidad de vida de la persona mayor es la salud. Se plantea como objetivo determinar si el nivel ocupacional de la persona mayor influye sobre su nivel de salud real. La muestra estuvo compuesta por 500 personas mayores de 60 años. El instrumento utilizado ha sido el Cuestionario de Conducta Ocupacional de los Mayores (C-COM). Existe una correlación moderada entre realizar un mayor número de ocupaciones y tener mayor nivel de salud. Estos resultados permiten orientar futuros programas y políticas ocupacionales. Además, si el estar ocupado mejora el nivel de salud, se podría reducir el gasto sanitario, invirtiendo en ocupación.

Palabras clave: Ocupación, Salud, Personas Mayores, Ocio, Calidad de Vida.

Influence of occupation on daily life and leisure activities on the health of the elderly

ABSTRACT: The 21st century is destined to conform by a population to trend to age. For this motive, strategies have to be looked to improve the quality of life of this sector of population. One of the variables that affect of more determinant form in the quality of life of the major person is the health. The aims of this study is to determinate if the occupational level of the elderly influences his health. The sample was composed by 500 sixty-year-old elderly. The used instrument has been the Questionnaire of Occupational Conduct of the Major (C-COM). A moderated correlation exists between realizing a major number of occupations and to have a major level of health. These results guide future programs and occupational policies. Also, if being busy improving the level of health could reduce health spending, investing in occupation.

Keywords: Occupation, Health, Elderly, Leisure, Quality of Life.

${ }^{1}$ Correspondencia: Universidad de Almería. Departamento de Psicología. La Cañada de San Urbano, s/n 04120. Almería (España). E-mail: jmaguilar@ual.es. 
El siglo XXI está destinado a conformarse por una población con tendencia a envejecer y en cuya estructura se consolidará la abundante presencia de personas mayores. Por este motivo, se han de buscar estrategias para la mejora de la calidad de vida de este sector de población. Una de las variables que inciden de forma más determinante en la calidad de vida de la persona mayor es la salud (Aguilar-Parra, Álvarez y Lorenzo, 2011; De Juanas, Limón y Navarro, 2013).

En este estudio se hipotetiza con la importancia de la ocupación como herramienta para mejorar la salud de las personas mayores y frenar los efectos propios del envejecimiento. Así la ONU en la Declaración Universal de Derechos Humanos recoge la importancia de que todas las personas tengan la posibilidad y la capacidad de ocuparse (ONU, 1948). Sin embargo, estos derechos están ausentes en muchas partes del mundo, tal y como señala Wilcock (2008), no disponen de esta posibilidad por la falta de salud y de dedicación a la ocupación, que pueden llevar al reconocimiento médico de la enfermedad física y mental, y a la discapacidad a largo plazo, sobre todo en las personas mayores.

Correa, Goldils, López y Pérez (2010) han señalado que desde la perspectiva terapéutica que la ocupación es fundamental para la adaptación humana, su ausencia o interrupción es una amenaza para la salud y cuando la enfermedad, el trauma o las condiciones sociales han afectado a la salud biológica o psicológica de una persona, la ocupación es un medio efectivo para reorganizar el comportamiento. La perspectiva preventiva se refiere al mantenimiento de la salud y calidad de vida a través del desarrollo de ocupaciones que favorezcan un alto nivel funcional y bienestar físico y mental.

El mayor, una vez jubilado, dispone de mayor cantidad de tiempo para el esparcimiento, puede dedicar más tiempo a sus ocupaciones favoritas y a sus hobbies. Tal y como señalan Butrica, Johnson y Zedlewski (2007, citado en Cole y Mcdonald, 2011) a medida que envejece la población, la demografía predice que muchos de los adultos mayores se pasan hasta 2530 años de una vida relativamente saludable después de su jubilación. Además la mayoría de las personas necesitan experimentar este sentimiento de utilidad y participación para mantener cierta sensación de pertenencia a algo, la sensación de poseer una vida emocional y sentimental realmente vivida desde y para la comunidad y de ser necesitadas, algo que sólo se consigue a través del rendimiento y la diligencia, o, si prefiere, como señalan importantes psicólogos como Piaget y Freud, la vida psíquica, su equilibrio y funcionamiento, no se mantiene sino a través de la "actividad" (Fernández-Lópiz, 1996).

Adams, Restorick y Cole (2011) valoran la importancia de la ocupación sobre la salud de la persona, resaltando la teoría de la actividad del envejecimiento que hacía hincapié en los beneficios de mantener un estilo de vida activo después de la jubilación, para remplazar los roles sociales que quedan atrás y desarrollar nuevas ocupaciones apropiadas para su edad.

Desde este enfoque, Moruno y Romero (2004) señalan que la ocupación se concibe como agente para la promoción de la salud y la prevención de la enfermedad, la discapacidad y el bienestar psicológico. La terapia ocupacional se basa en la creencia de que existe una relación entre la ocupación, la salud y el bienestar (Law, Steinwendner, y Leclair, 1998, citado en Creek y Hughes, 2008). Asimismo, Sáez-Narro, Aleixandre y Meléndez (1994) y Vega, Buz y Bueno (1988) señalaron que se ha de partir de que el ocio y la actividad en la tercera edad, son una parte obligada para una vejez productiva y sana. 
En esta línea, Hakansson, Lissner, Bjrkelund y Sonn (2009) estudiaron el nivel de ocupación diaria (trabajo remunerado, trabajo doméstico y ocupación de ocio) de las mujeres en edad de trabajar en relación con su nivel de salud percibida. Asimismo, la ocupación en tareas del quehacer diario es fundamental para mantener las capacidades, muchas personas mayores planifican su jornada cotidiana y le proporcionan de este modo cierto ritmo, garantizando las capacidades mentales, cognitivas, sociales y físicas (Álvarez y Sicilia, 2007; Álvarez, Aguilar-Parra y Mercader, 2012; Fernández-Lópiz, 1996).

Morales y Bravo (2006) señalan que también el ocio tiene un carácter beneficioso que forma parte de la naturaleza intrínseca del ocio. Conciben el ocio terapéutico como un proceso que utiliza los servicios de ocio para promover cambios deseables en el aspecto físico, emocional o social de la conducta del individuo y favorecer su desarrollo personal. Diversos autores han, demostrado que participar en ocupaciones de ocio conllevaría un aumento del bienestar físico, de la salud mental, del funcionamiento social, autonomía funcional (Yarmohammadian y Yazdkhasti, 2010), o de todas ellas (Ball, Corr, Knight, y Lowis, 2007). García-Martín (2002) señala que gran parte de los estudios que se han centrado en los beneficios psicológicos derivados de la participación en actividades de ocio en los mayores, han aportado resultados satisfactorios. Así, se ha comprobado que una mayor implicación en este tipo de actividades se traduce en niveles de bienestar subjetivo más alto (Brown, Frankel y Fennell, 1991; Dupuis y Smale, 1995), disminución del sentimiento de soledad (Lee e IshiiKuntz, 1987; Mullins y Mushel, 1992), aumento del estado de ánimo (Lee e Ishii-Kuntz, 1987; Tinsley, Colbs, Treaf y Kaufman, 1987) o incremento en la capacidad de afrontamiento de los cambios producidos durante el envejecimiento (Kelly, Steinkamp y Kelly, 1987; Steinkamp y Kelly, 1985). Lo que confirma la afirmación de Argyle (1996): "El ocio es una fuente muy importante de bienestar, sobre todo para los que no están trabajando, y más importante que el trabajo para muchos que lo están”.

Se sabe que determinados tipos de actividad, como la actividad física, tienen efectos beneficiosos para la salud. Investigadores como DiPietro (2001) o Dutra, Oliveira, Lemos, Bezerra y Drummond (2011) han descrito los cambios en el patrón de actividad física en la vejez y la relación de estos cambios con la autonomía funcional de los sujetos. Según sus estudios, niveles moderados de actividad física protegen de determinadas enfermedades crónicas o de los efectos negativos de éstas. Además, se pueden conseguir efectos notablemente beneficiosos para la salud independientemente de la buena forma física que podría obtenerse con una actividad de gran intensidad. Es decir, actividades moderadas como andar, subir escaleras, pasear en bici o cuidar el jardín, tienen efectos muy positivos sobre el incremento de la energía vital y el mantenimiento del tono muscular.

Demostrando la importancia de la ocupación, Adams et al. (2011) señalaron que la reducción de la participación social por la desocupación, puede desembocar en depresión y un empeoramiento del estado de salud. Las personas con depresión reducían su nivel de actividad, empeorando su autonomía y su salud, aumentando su mortalidad (Manini. et al., 2006). Además, el efecto de la ocupación parece ser un factor determinante de la salud cognitiva, psicosocial y emocional, pudiendo ser un factor de prevención del deterioro cognitivo (Clark et al., 2012; Hendrie et al., 2006). 
La justificación de este estudio es debido a la importancia de la ocupación sobre la salud de las personas mayores, como estrategia para la mejora de la calidad de vida y del bienestar de este sector de población. El objetivo de esta investigación es determinar si el nivel ocupacional de la persona mayor de 60 años influye sobre su salud real. Según la literatura previa, se puede hipotetizar que el desarrollar un mayor número de ocupaciones, permite a la persona posea un mejor nivel de salud, que aquellas personas que realiza un menor número de ocupaciones y son más sedentarias, sobre todo después de la jubilación.

\section{Método}

\section{Diseño}

El diseño de la presente investigación es un estudio observacional, descriptivo, de corte transversal y correlacional, de la calidad de vida relacionada con la salud de los mayores que se ocupan en determinadas actividades en la capital almeriense y en su provincia.

\section{Participantes}

La muestra utilizada en el presente estudio, estuvo compuesta por un total de 500 personas mayores de 60 años de ambos sexos, de Almería y su provincia (279 mujeres y 221 hombres). La media de la edad de la muestra fue de $\bar{x}=70,80$ años, con una desviación típica de (S.D.=8,110). El método de muestreo que se ha utilizado es el muestreo aleatorio estratificado por afijación proporcional (según el municipio de residencia, atendiendo a todas las comarcas o zonas de la provincia y a la capital). Las variables de segmentación a estratificar fueron la edad y género (6 grupos de edad x 2 sexos=12 estratos). Para la edad se han utilizado 6 estratos diferentes que abarcan desde los 60 años en adelante (60-64; 65-69; 70-74; 75-79; 80-84; 85 y más años).

\section{Instrumentos}

Para la obtención de datos, se utilizó el Cuestionario de Conducta Ocupacional de los Mayores (C-COM) de Aguilar (2012), que está compuesto por 7 ítems, que tratan de extraer la conducta ocupacional de los mayores, tanto en las actividades de la vida diaria como en su tiempo de ocio (cómo viven, cuál es su nivel de salud y de autonomía, etc.). Es una herramienta que se construyó "ad hoc", por lo que es un instrumento de recogida de información que no arroja puntuaciones globales, sino únicamente describe la posición de la persona frente a los distintos interrogantes que se plantean en el cuestionario (no se encontraron otros instrumentos que midieran lo que se pretendía y no obstante, únicamente lo que se pretende es recoger información sobre las ocupaciones que realiza la persona, cómo se percibe en relación a su estado de salud y cuál es el número de visitas al médico que realiza habitualmente y su consumo farmacológico). El cuestionario fue validado interjueces obteniendo una buena fiabilidad y consistencia de las puntuaciones otorgadas por los jueces mediante el coeficiente de Cronbach y el estadístico W de Kendall. Asimismo fue validado en estudio piloto con 183 sujetos (Aguilar, 2012) mostrando una excelente discriminación en la validez concurrente con 
otros cuestionarios de salud (SF-36, referido a los ítems de salud del cuestionario) y autonomía (Índice de Barthel).

\section{Procedimiento}

Se tomaron las medidas necesarias a 700 sujetos de los cuales aleatoriamente se han seleccionado 500 únicamente de los cuestionarios que poseían todos los datos correctamente interpretados. Tras la aplicación del cuestionario C-COM, se procedió a tabular las puntuaciones en el paquete informático SPSS (Statistical Package for Social Sciencies), en su versión 20.0, para finalmente desarrollar este informe con los datos recogidos.

\section{Análisis de datos}

Se han utilizado las tablas de contingencia, apoyadas por el estadístico $\chi^{2}$ para determinar el nivel de dependencia o relación entre variables y la $|\mathrm{r}|$ y la Tau-C para valorar el tamaño del efecto de una variable sobre la otra. Se ha trabajado con los datos obtenidos en los ítems referidos a salud real del cuestionario C-COM (número de visitas al médico y consumo farmacológico).

\section{Resultados}

Comprobar si existe relación entre la salud real de las personas de la muestra y su nivel de ocupación, tanto de ocupaciones del quehacer diario como ocupaciones de ocio.

En relación a las variables "número de visitas al médico" y la variable "ocupaciones del quehacer diario", se encuentra una relación significativa a través del estadístico $\chi^{2}(36, N=$ $499)=148,994 ; p<.001$ ), indicando que estas dos variables son dependientes. Lo mismo ocurre en relación a las variables "número de visitas al médico" y la variable "ocupaciones de ocio", se encuentra una relación significativa a través del estadístico $\chi^{2}(84, N=499)=$ 237,196; $p<.001$ ), por lo que estas dos variables también son dependientes. Finalmente se ha contrastado el nivel de ocupación total (tareas del quehacer diario más ocupaciones de ocio) con el número de visitas al médico y también se ha hallado relación significativa $\chi^{2}(108, N=$ $499)=305,694 ; p<$. 001). Se ha contrastado el nivel de correlación entre estas variables, obteniéndose una $|\mathrm{r}|=-.398$ en el caso de las ocupaciones del quehacer diario y el número de visitas al médico, una $|\mathrm{r}|=-.506$ en el caso de la ocupación de ocio, y una $|\mathrm{r}|=-.504$ en el caso de la ocupación total. Por lo que se halla una correlación moderada en el caso de la ocupación en tareas del quehacer diario y alta en relación a las ocupaciones de ocio. El coeficiente de correlación es de tipo indirecto en todos, por lo que cuando crece una variable la otra disminuye, es decir, a mayor nivel ocupacional (a más ocupaciones del quehacer diario o de ocio desempeñadas por la persona) menos visitas se producen al médico.

Para conocer el grado de dependencia entre estas variables, se ha desarrollado el estadístico inferencial coeficiente $(\mathrm{Tau}-\mathrm{C})$, con un resultado de $\mathrm{Tau}-\mathrm{C}=-.315$ en el caso de las ocupaciones del quehacer diario, Tau $-C=-.420$ en las ocupaciones de ocio, y Tau $-C=-.422$ en el caso de ocupación total. Por lo que se puede afirmar que existe una asociación moderada (pudiera ser alta dependiendo de la estimación que se considere) entre asistir un número de veces al médico y tener un determinado nivel de ocupación tanto en tareas del quehacer diario como en ocupaciones de ocio. 
Atendiendo a los residuos tipificados corregidos, en relación a las ocupaciones del quehacer diario, se percibe un mayor porcentaje del esperado de personas que van menos de 6 veces al año al médico y que realizan bastantes ocupaciones del quehacer diario, por ejemplo, las personas que realizan 6 (25,8\%; $Z=2.8)$ ó 7 (19\%; $Z=3.2)$ ocupaciones cada día. Por el contrario, se aprecia que las personas que no realizan ocupaciones o realizan muy pocas, presentan porcentajes muy altos en el número de visitas al médico. Por ejemplo, quien va tres veces o más al mes y no realiza ninguna ocupación $(16,9 \% ; Z=6.4)$ o realiza una $(27,7 \% ; Z=$ 4.6). Mediante una visión general de los residuos tipificados, se aprecia como las personas que presentan un mayor índice de ocupación en tareas del quehacer diario muestran un número de visitas al médico menor que va aumentando gradualmente conforme disminuye el nivel de ocupación de la persona.

Atendiendo a los residuos tipificados corregidos, en relación a las ocupaciones de ocio desarrolladas por la persona, se percibe como se encuentra un mayor porcentaje del esperado de personas que acuden menos de seis veces al año al médico y que realizan bastantes ocupaciones de ocio (por ejemplo, las personas que realizan 19 ó 20 ocupaciones y acuden menos de seis veces al año a la consulta médica, representan el 6,7\%; $Z=3.7$ y $2,5 \% ; Z=2.5$ respectivamente). Por el contrario, se observa que acude tres veces o más al mes al médico, la persona que realiza sólo una ocupación $(18,5 \% ; Z=5.9)$ o realiza dos $(26,2 \% ; Z=3.5)$. Mediante una visión general de los residuos tipificados, se aprecia como las personas que presentan un mayor índice de ocupación de ocio muestran un menor número de visitas al médico que va aumentando gradualmente conforme disminuye el nivel de ocupación de la persona.

En relación a las variables "nivel de consumo farmacológico" y la variable "ocupaciones del quehacer diario", se encuentra una relación significativa a través del estadístico $\chi^{2}(36, N=$ $499)=110,495 ; p<.001$ ), lo que indica que estas dos variables son dependientes. Lo mismo ocurre en relación a las variables "nivel de consumo farmacológico" y la variable "ocupaciones de ocio", se encuentra una relación significativa a través del estadístico $\chi^{2}(84, N=499)=$ 232,$811 ; p<.001$ ), lo que indica que estas dos variables también son dependientes. Finalmente se ha contrastado el nivel de ocupación total (tareas del quehacer diario más ocupaciones de ocio) con el consumo farmacológico y también se ha hallado relación significativa $\chi^{2}(108, N=$ $499)=282,731 ; p<.001)$. Además, se ha contrastado el nivel de correlación entre ambas variables, obteniéndose una $|\mathrm{r}|=-.345$ en el caso de las ocupaciones del quehacer diario y el nivel de consumo farmacológico, una $|\mathrm{r}|=-.481$ en el caso de la ocupación de ocio, y una $|\mathrm{r}|$ $=-.479$ en el caso de la ocupación total. Por lo que se halla una correlación moderada en todos los casos. El coeficiente de correlación es de tipo indirecto en los estadísticos, por lo que cuando crece una variable la otra disminuye, es decir, a mayor nivel ocupacional (a más ocupaciones del quehacer diario o de ocio desempeñadas por la persona) menos consumo farmacológico.

Para conocer el grado de dependencia, entre estas variables se han utilizado el estadístico inferencial coeficiente $(\mathrm{Tau}-\mathrm{C})$, con un resultado de $\mathrm{Tau}-\mathrm{C}=-.287$ en el caso de las ocupaciones del quehacer diario, Tau-C $=-.404$ en las ocupaciones de ocio, y Tau $-C=-.394$ en el caso de ocupación total. Por lo que se puede afirmar que existe una asociación baja, casi 
moderada entre el número de fármacos que se consumen y tener un determinado nivel de ocupación en tareas del quehacer diario y una asociación moderada en relación a las ocupaciones de ocio.

Atendiendo a los residuos tipificados corregidos, en relación a las ocupaciones del quehacer diario desarrolladas por la persona, se percibe como se encuentra un mayor porcentaje del esperado de personas que no toman medicación o toman menos pastillas y que realizan bastantes ocupaciones del quehacer diario. Por ejemplo, las personas que realizan 7 ocupaciones y no toman medicación $(22,3 \% ; Z=3.7)$ ó los que sólo toman una pastilla al día $(6,8 \% ; Z=2.6)$ y realizan 8 ocupaciones del quehacer diario cada día. Por el contrario, se aprecia que las personas que no realizan ocupaciones o realizan muy pocas, presentan porcentajes muy altos en el consumo de fármacos diario, por ejemplo, quien toma 4 o más fármacos al día y no realiza ninguna ocupación $(8,1 \% ; Z=4)$ o realiza una $(20,6 \% ; Z=4.7)$. Mediante una visión general de los residuos tipificados, se aprecia como las personas que presentan un mayor índice de ocupación en tareas del quehacer diario muestran un consumo farmacológico menor que va aumentando gradualmente conforme disminuye el nivel de ocupación de la persona.

Atendiendo a los residuos tipificados corregidos, en relación a las ocupaciones de ocio desarrolladas por la persona, se percibe como se encuentra un mayor porcentaje del esperado de personas que no toman medicación y que realizan bastantes ocupaciones de ocio. Por ejemplo, las personas que realizan 19 ó 20 ocupaciones y no toman medicación (representan el 10,7\%; $Z$ $=5.8$ y 2,7\%; $Z=3.2$ respectivamente). Por el contrario, se aprecia que las personas que no realizan ocupaciones o realizan muy pocas, presentan porcentajes muy altos pero en este caso en el consumo de fármacos diario, por ejemplo, quien toma 4 o más fármacos al día y realiza sólo una ocupación $(10,6 \% ; Z=4.6)$ o realiza dos $(28,8 \% ; Z=7.5)$. Mediante una visión general de los residuos tipificados, se aprecia como las personas que presentan un mayor índice de ocupación de ocio muestran un consumo farmacológico menor que va aumentando gradualmente conforme disminuye el nivel de ocupación de la persona.

\section{Discusión}

En cuanto a la relación del número de visitas al médico (salud real) con el nivel de ocupaciones del quehacer diario que desarrolla la persona, según los resultados obtenidos se puede afirmar que son dependientes. Los mismos resultados se obtienen en relación con las ocupaciones de ocio. Por lo tanto, atendiendo a las correlaciones, se puede concluir que a mayor nivel ocupacional menos visitas se producen al médico. Se aprecia como las personas que presentan un mayor índice de ocupación en tareas del quehacer diario o en ocupaciones de ocio, muestran un número de visitas al médico menor que va aumentando gradualmente conforme disminuye el nivel de ocupación de la persona. Esta persona está más sana, resultados coinciden con los recogidos previamente en algunas investigaciones que mantienen que la ocupación mejora la salud real de la persona (Adams et al., 2011; Ball et al., 2007; Carmel, Morse y Torres-Gil, 2007; Cole y Mcdonald, 2011; Correa et al., 2010; Morales y Bravo, 2006; Romero y Moruno, 2003 citado en Bellido, Berrueta y Ariñez, 2004; entre otros). 
Se verifica que necesitar menos asistencia médica, se relaciona con estar más ocupado, el motivo de que la ocupación contribuya a reducir el número de visitas al médico, está claramente relacionado con que la persona se siente y poseen un mejor estado de salud físico y mental, por lo que no requiere de asistencia médica o al menos, no de forma asidua. Morales y Bravo (2006) apuntan que la ocupación de ocio tiene efectos muy beneficiosos sobre la salud física y mental, promueve cambios deseables en el aspecto físico, emocional o social de la conducta del individuo y favorece su desarrollo personal, además de utilizarse para superar o mejorar un problema o situación que afecta negativamente al ocio de esa persona (superar una adicción, recuperarse de algún trastorno físico o psicológico...). Viana, García-Pinto y Ávila (2003) señalan que en nuestra sociedad y en este momento, la mayoría de los seres humanos realizan estas actividades de forma automática, sin pararse a analizar el complejo entramado y la buena integración de un gran número de componentes ocupacionales involucrados para su adecuada realización (componentes físico, sensoriales, cognitivos y psicosociales). Se puede decir que esta área solamente es valorada por el individuo, en su real dimensión, cuando por alguna circunstancia se pierde o se disminuye la posibilidad de realizar las actividades de la vida diaria de manera independiente (disminución de movilidad, dolor, pérdida de memoria, falta de impulso vital, etc.). Desde la prevención, se debe de concienciar al individuo sobre sus acciones, sus ritmos naturales, la importancia del cuidado personal para su propia autoestima y la interacción con el medio social. Y, en los casos en los que se haya perdido la independencia en dichas actividades, se buscará la recuperación de las mismas.

La ocupación favorece un mejor estado de salud, por lo que una persona que está más sana, consume menos medicamentos. Si se tiene en cuenta la importancia de reducir el gran consumo farmacológico que presentan las personas mayores, tal y como destacaban en su estudio Peña, Redondo y Groning (2003) que encontraron que el 81 \% de los ancianos consumen algún fármaco (exceptuando vitaminas, contabilizando estas sería un porcentaje mayor), siendo el motivo principal de prescripción las afecciones cardiovasculares y los dolores articulares, este consumo es excesivamente alto. Además, una importante proporción de fármacos consumidos por los ancianos son poco eficaces para las indicaciones teóricas a las que están destinados (Pérez, 1995; Samsa et al., 2004), ya que todavía existen pocos tratamientos curativos de las enfermedades crónicas que afectan a las personas ancianas, siendo la terapéutica normalmente sintomática. Este motivo conduce al uso y consumo de múltiples fármacos que, debido a las especiales condiciones farmacocinéticas y farmacodinámicas de estos pacientes, producen un elevado número de reacciones adversas (Lázaro y Ribera, 1994; Pérez, 1995; Willcox, Himmelstein y Woolhandler, 1994) que están en relación directa con el número de fármacos consumidos (Nolan y O’Malley, 1998). Varios autores (Gavilán-Moral, Villafaina-Barroso, Jiménez-de Gracia y Gómez, 2011; Pedrera et al., 1999), afirman que la dimensión de esta problemática es mucho mayor, por las graves repercusiones socio-sanitarias que conlleva, ya que se produce una elevación del gasto en recursos sanitarios respecto a otros grupos de población, hecho este que se pone de manifiesto, el que más de 1/3 de las consultas a demanda en Atención Primaria procede de personas mayores de 65 años. Por ello queda patente la importancia de reducir el consumo farmacológico de los mayores, mejorando su salud a través de la ocupación, de forma que se reduzca el gasto sanitario y se reduzcan los efectos secundarios de los fármacos sobre su salud. 
Como conclusión en relación al número de visitas al médico y el número de pastillas que se toman al día (consumo farmacológico), valorándolos como criterio de salud personal, se han hallado correlaciones entre estas variables y el nivel de ocupación. Las correlaciones son moderas y altas, pero cabe destacar que podrían ser mayores, ya que se ha percibido una limitación en la herramienta de medición del ítem referido al número de pastillas que se consume, ya que se ha codificado en ninguna, una, dos, tres, cuatro o más, cuando la mayoría de las personas han seleccionado que toman 4 o más, aclarando algunas personas que eran más de 14, por lo que se deberían haber codificado en rangos mayores, para establecer de forma más contundente la diferencia entre consumos farmacológicos de las personas más ocupadas. No obstante, ya aparecen unas correlaciones moderadas e incluso altas entre estas variables entre el nivel de ocupación y el consumo farmacológico, por lo que posiblemente, con una corrección del ítem, la correlación habría sido más alta. En relación a las visitas al médico, parece comprobarse que también existe una correlación moderada, que puede estar debida a que las personas necesitamos de un control médico periódico. Si la ocupación de la persona redunda en una mejor salud percibida, las visitas al médico y el consumo farmacológico será menor en este caso, puesto que si nos percibimos con un buen estado de salud, necesitaremos hacer un menor uso de estos servicios del sistema nacional de salud. El antecedente de este trabajo, sobre la posibilidad de reducir el gasto sanitario y social a través de la ocupación de la persona mayor, se realiza en el "Estudio sobre el Buen Envejecimiento" (Well Elderly Study 1, Jackson, Carlson Mandel, Zemke y Clark, 1995 y Study 2, Clark et al., 2012), que proporcionó una prueba científica de la efectividad de la ocupación en la modificación del estilo de vida y ocupaciones de las personas mayores que viven en su entorno habitual. Tal y como recoge Cole (2011) se mejoró su salud física y mental, superando las barreras que le impedían participar en actividades de la vida diaria y en las relaciones con los demás. Los datos muestran que la intervención es rentable económicamente según un estudio publicado en la Revista de la Sociedad Americana de Geriatría (Journal of the Americans Geriatrics Society). Este estudio es el primero en la historia de la T.O. en utilizar las metodologías más modernas de investigación que provienen del campo de la economía de la salud. A través de entrevistas diseñadas para descubrir cuáles eran los gastos que la persona mayor generaba relacionados con su salud, se descubrió que los mayores que se beneficiaron de un tratamiento de T.O. ocasionaron menores gastos en otros servicios de salud. De hecho, el ahorro promedio supera el coste de la propia terapia, lo que sugiere que la T.O. y la terapia a través de la ocupación, puede ahorrar dinero a los servicios sanitarios, mejorando además de la salud, su calidad de vida. En otro estudio realizado por Aparicio, Carbonell-Baeza y Delgado-Fernández (2010) advierten que invertir en ejercicio físico u ocupaciones de ocio activo de calidad para la población mayor a través de profesionales cualificados, podría derivar en un ahorro considerable para la sanidad, tanto pública como privada. Además en esta línea, Sergeant (1995) señalaron que los programas dirigidos a la tercera edad, debían ir dirigidos a evitar la pérdida de movilidad, que conllevaban la pérdida de independencia, contribuyendo a una mayor demanda de servicios sanitarios, al aislamiento social, depresión y abandono. En este sentido apuntan los resultados obtenidos en este estudio, en la que estos se orientan hacia una correlación significativa entre salud y reducción del gasto farmacológico, de visitas al médico, de niveles de dolor, de mejor salud mental, etc. por lo que se muestran indicios claros de los beneficios de las ocupaciones sobre la salud de la persona y el menor gasto en salud y de forma directa en servicios sociales, ya que 
una persona que se mantiene por un mayor periodo de tiempo con una mayor independencia y autonomía, no necesitará apoyo por parte de las instituciones públicas sociales. En un estudio que se está realizando con personas mayores que realizan programas ocupacionales, y en los que se han realizado pre test y post test, y se han computado todos y cada uno de los fármacos que consume cada persona, las visitas al médico realizadas y la salud real de la persona, parecen apuntar a una reducción clara del consumo farmacológico, de las visitas al médico y una mejora sustancial de su salud real que se confirmarán al finalizar el estudio. No obstante, este trabajo abre un camino importante de reducción del gasto sanitario y social, que será imprescindible definir claramente para contribuir a mejorar el gasto en estos momentos de austeridad y aumentar el nivel de calidad de vida de la persona mayor.

\section{Limitaciones}

Entre las limitaciones halladas, en el cuestionario "ad hoc" C-COM, posee en uno de sus ítems (cuántas pastillas toma la persona al día, y se recogen como posibles respuestas 1, 2, 3, 4 o más). Se ha detectado que la mayoría de las personas de la muestra tienen una medicación muy elevada, por lo que lo ideal hubiera sido recoger rangos más amplios, ya que no es posible comparar a una persona que toma 4 pastillas al día con casos de 14. Esto, posiblemente, hubiera mejorado los resultados que hemos obtenido y hubiesen aumentado las correlaciones, obteniendo resultados mucho más positivos. También hubiese sido interesante recoger la cantidad exacta y el nombre del fármaco.

\section{Referencias}

Adams, K. B., Restorick, A. \& Cole, M. B. (2011). Changes in activity and interest in the third and fourth age: Associations with health, functioning and depressive symptoms. Occupational Therapy International, 18(1), 4-17.

Aguilar, J. M. (2012). La conducta ocupacional, el proceso de toma de decisiones y su influencia en la calidad de vida de los mayores. (Tesis Doctoral sin publicar). Universidad de Almería. Almería, España.

Aguilar-Parra, J. M., Álvarez, J. \& Lorenzo, J. J. (2011). Factores que determinan la calidad de vida de las personas mayores. International Journal of Developmental and Educational Psychology, 1(4), 161-168.

Álvarez, J., Aguilar-Parra, J. M. \& Mercader, I. (2012). Cognición y autonomía en personas mayores dependientes. Anales de Psicología, 28(3), 945-952.

Álvarez Hernández, J., \& Sicilia Molina, M. (2007). Deterioro cognitivo y autonomía personal básica en personas mayores. Anales de Psicología, 23(2), 272-281.

Aparicio, V. A., Carbonell-Baeza, A. \& Delgado-Fernández, M. (2010). Beneficios de la actividad física en personas mayores. Revista Internacional de Medicina y Ciencias de la Actividad Física y el Deporte, 10(40), 556-576.

Argyle, M. (1996). The social psychology of leisure. Nueva York: Penguin Books.

Ball, V., Corr, S., Knight, J. \& Lowis, M. J. (2007). An investigation into the leisure occupations of older adults. The British Journal of Occupational Therapy, 70(9), 393400. 
Bellido, J. R., Berrueta, L. M. \& Ariñez, I. (2004). La aportación de la terapia ocupacional en el abordaje de la funcionalidad del trastorno de la personalidad. Revista Gallega de Terapia Ocupacional, 1. Recuperado de http://revistatog.3owl.com/num1/pdfs/num1art6.pdf.

Brown, B. A., Frankel, B. G. \& Fennell, M. (1991). Happiness through leisure: The impact of type of leisure activity, age, gender and leisure satisfaction on psychological wellbeing. Journal of Applied Recreation Research, 16, 367-391.

Carmel, S., Morse, C. \& Torres-Gil, F. (2007). Lessons on aging from three nations. The art of aging well. New York: Amityville.

Clark, F., Jackson, J., Carlson, M., Chou, C., Cherry, B., Jordan-Marsh, M.... Azen, S. P. (2012). Effectiveness of a lifestyle intervention in promoting the wellbeing of independently living older people: Results of the well elderly 2 randomised controlled trial. Journal of Epidemiology \& Community Health, 66(9), 782-790.

Cole, M. B. (2011). Special Issue on Occupational Therapy in the Third Age. Occupational Therapy International, 18(1), 1-3.

Cole, M. B. \& Macdonald, K. C. (2011). Retired occupational therapists' experiences in volunteer occupations. Occupational Therapy International, 18(1), 18-31.

Correa, M., Goldils, M. Y. López, L. \& Pérez, C. (2010). La Ocupación Dentro de la Terapia Ocupacional. Revista Electrónica de Terapia Ocupacional, 5. Recuperado de http://espaciotovenezuela.com/art_abril2010/ocupacion_dentro_terapiaocupacional.pdf

Creek, J. \& Hughes, A. (2008). Occupation and health: A review of selected literature. British Journal of Occupational Therapy, 71(11), 456-458.

De Juanas, A., Limón, M. R. \& Navarro, E. (2013). Análisis del bienestar psicológico, estado de salud percibido y calidad de vida en personas adultas mayores. Pedagogía Social. Revista Interuniversitaria, 1(22), 153-168.

DiPietro, L. (2001). Physical activity in aging: Changes in patterns and their relationship to health and function. Journal of Gerontology: Series A: Biological Sciences and Medical Sciences, 56a(11), 13-22.

Dupuis, S. L. \& Smale, B. J. (1995). An examination of relationship between psychological well-being and depression and leisure activity participation among older adults. Society and Leisure, 18(1), 67-92.

Dutra, F., Oliveira, W., Lemos, H., Bezerra, E. \& Drummond, E. (2011). Functional autonomy of physically active and sedentary elderly women: comparative causal study. Brazilian Journal of Nursing, 10(3), 1-10.

Fernández-Lópiz, E. (1996). Tiempo libre y nuevas responsabilidades en los adultos mayores. Propuestas: Revista de la Universidad Nacional de la Matanza, 2(4), 31-44.

García-Martín, M. A. (2002). La actividad y el ocio como fuente de bienestar durante el envejecimiento. Efdeportes: Revista Digital. Recuperado de http://www.efdeportes.com /efd47/bien.htm.

Gavilán-Moral, E., Villafaina-Barroso, A., Jiménez-de Gracia, L. \& Gómez, M. C. (2012). Ancianos frágiles polimedicados: ¿es la deprescripción de medicamentos la salida? Revista Española de Geriatría y Gerontología, 47(4), 162-167.

Håkansson, C., Lissner, L., Björkelund, C. \& Sonn, U. (2009). Engagement in patterns of daily occupations and perceived health among women of working age. Scandinavian Journal of Occupational Therapy, 16(2), 110-117. 
Hendrie, H. C., Albert, M. S., Butters, M. A., Gao, S., Knopman, D. S., Launer, L. J., Yaffe, K., ..., Wagster, M. V. (2006). The NIH Cognitive and Emotional Health Project. Alzheimer's and Dementia, 2(1), 12-32.

Jackson, J., Carlson, M., Mandel, D., Zemke, R., \& Clark, F. (1998). Occupation in lifestyle redesign: The Well Elderly Study Occupational Therapy Program. American Journal of Occupational Therapy, 52(5), 326-336.

Kelly, J. R., Steinkamp, M. W. \& Kelly, J. R. (1987). Later-life satisfaction: Does leisure contribute? Leisure Sciences, 9, 189-200.

Lázaro, M. \& Ribera, J. M. (1994).Tratamiento del paciente anciano con problemas médicos múltiples. Informes Terapia Sistema Nacional de Salud, 18, 109-119.

Lee, G. R. \& Ishii-Kuntz, M. (1987). Social interaction, loneliness, and emotional well-being among the elderly. Research on Aging, 9, 459-482.

Manini, T. M., Everhart, J. E., Patel, K. V., Schoeller, D. A., Colbert, L. H., Visser, M., ..., Harris, T. B. (2006). Daily activity energy expenditure and mortality among older adults. Journal of the American Medical Association, 296, 171- 179.

Morales, M. \& Bravo, I. (2006). El ocio terapéutico. En Giró. Envejecimiento activo, envejecimiento en positivo. Logroño: Universidad de La Rioja. Servicio de Publicaciones.

Moruno, P. \& Romero, D. M. (2004). Terapia Ocupación en salud mental: la ocupación como entidad, agente y medio de tratamiento. Revista Gallega de Terapia Ocupacional, 1, 1-18. Extraído el 18 de junio de 2009 desde http://www.revistatog.com/num1.htm.

Mullins, L. C. \& Mushel, M. (1992). The existence and emotional closeness of relationships with children, friends and spouses: the effect on loneliness among older persons. Research on Aging, 14(4), 448-47.

Nolan, L. \& O'Malley, K. (1998). Precribing for the elderly. Adverse drug reaction in the elderly. Journal of American Geriatric Society, 36,142-149.

ONU (1948). Declaración Universal de los Derechos Humanos. Recuperado de http://www.un.org/es/documents/udhr/.

Pedrera, J. D., Canal, M. L., Lavado, J. M., Postigo, S., Sánchez-Belda, M. \& Durán, N. (1999). Estudio de salud de las personas mayores en Extremadura: Consumo de fármacos y patologías crónicas más frecuentes. Revista Española de Salud Pública, 73(6), 677-686.

Peña, M. A., Redondo, A. \& Groning, E. (2003). Consumo de medicamentos en ancianos. Revista Cubana Medicina General Integral, 19(3). Recuperado de http://bvs.sld.cu/revistas/mgi/vol19_3_03/mgi07303.htm.

Pérez, A. (1995). Enfermería Geriátrica. Madrid: Editorial Síntesis.

Sáez-Narro, N. Aleixandre, M. \& Meléndez, J. C. (1994). Actividad y tiempo libre en la tercera edad. Revista de Psicología de la Educación, 14, 5-24.

Samsa, G. P., Hanlon, J. T., Schmader, K. E., Weinberger, M., Clipp, E. C., Utretch, K. M.,..., Cohen, H. J. (1994). A summated score for the medication appropriateness index: development and assessment of clinimetric properties including content validity. Journal Clinical Epidemiology, 47, 891-896.

Sergeant, A. J. (1995). Human muscle function. Age related changes and adaptations to activity programmes in the elderly. Amsterdam: Vrije University. 
Steinkamp, M. W. \& Kelly, J. R. (1985). Relationships among motivation orientation, level of leisure activity and life satisfaction in older men and women. The Journal of Psychology, 119, 509-520.

Tinsley, H. E., Colbs, S. L., Treaf, J. D. \& Kaufman, N. (1987). The relationship of age, gender, health and economic status to the psychological benefits older persons report from participation in leisure activities. Leisure Sciences, 9, 53-65.

Vega, J. L. (1988). Modelo de seguimiento y evaluación del programa de educación de adultos. Salamanca: Diputación de Salamanca.

Vega, J. L., Buz, J. \& Bueno, B. (2002). Niveles de actividad y participación social en las personas mayores, Revista Interuniversitaria de Formación del Profesorado, 45, 33-53.

Viana, I., García-Pinto, M. C. \& Ávila, A. (2003). Ocupación Alienante Versus Ocupación Significativa. Recuperado de www.terapia-ocupacional.com.

Wilcock, A. (2008). Professional Identity. Revista Terapia Ocupacional Galicia, 2, 35-47. Recuperado de http://revistatog.3owl.com/mono/num2/wilcock.pdf.

Willcox, S. M., Himmelstein, D. U. \& Woolhandler, S. (1994). Inappropiate drug prescribing for the comunity-dwelling elderly. JAMA, 272, 292-296.

Yarmohammadian, A. \& Yazdkhasti, F. (2010). The effect of the new occupation on the mental health of retired persons. Studia Psychologica, 52(1), 69-76. 